\title{
LINEAR ORBIT PARAMETERS FOR THE EXACT EQUATIONS OF MOTION*
}

\section{G. Parzen, Brookhaven National Laboratory, Upton, NY 11973 USA}

\section{Abstract}

This paper defines the beta function and other linear orbit parameters using the exact equations of motion. The $\beta, \alpha$ and $\psi$ functions are redefined using the exact equations. Expressions are found for the transfer matrix and the emittance. The differential equations for $\eta=x / \beta^{1 / 2}$ is found. New relationships between $\alpha, \beta, \psi$ and $\nu$ are derived.

\section{INTRODUCTION}

This paper defines the beta function and the other linear orbit parameters using the exact equations of motion. The usual treatment [1] of the linear orbit parameters is based on the approximate equation of motion

$$
\frac{d^{2} x}{d s^{2}}+K(s) x=0
$$

Approximations are made in obtaining Eq. (1) which are usually valid for large accelerators.

The exact linearized equations of motion can be written as

$$
\begin{aligned}
\frac{d x}{d s} & =A_{11} x+A_{12} p_{x} \\
\frac{d p_{x}}{d s} & =A_{21} x+A_{22} p_{x}
\end{aligned}
$$

$x$ and $p_{x}$ are the canonical coordinates in a curvilinear coordinate system based on a reference orbit and the $A_{i j}(s)$ are periodic in $s$ with period $L$. The approximate Eq. (1) assumes that $A_{11}=A_{22}=0, A_{12}=1$ and $A_{21}=-K(s)$. The exact values of the $A_{i j}$ are given in [2].

A treatment of the linear orbit parameters based on the exact equations, Eqs. (2), rather than the approximate Eq. (1) may be desirable in the following situations:

1. Symplectic long term tracking using a procedure where the magnets are replaced by a sequence of point magnets and drift spaces. For the tracking to be symplectic, one has to use the solutions of the exact equations of motion. The linearized equations of motion then have the form of Eq. (2).

2. Small accelerators where the approximations made in deriving Eq. (1) may not be valid.

Many of the results found using the approximate equations carry over for the exact equations. A few of the changed results are the following:

$$
\begin{aligned}
\alpha & =\frac{1}{A_{12}}\left(-\frac{1}{2} \frac{d \beta}{d s}+A_{11} \beta\right) \\
\psi & =\int_{0}^{s} A_{12} \frac{d s}{\beta}
\end{aligned}
$$

\footnotetext{
*Work performed under the auspices of the U.S. Department of Energy.
}

$$
v=\frac{1}{2 \pi} \int_{0}^{C} d s \frac{A_{12}}{\beta}
$$

where $C$ is the circumference of the accelerator.

\section{EIGENFUNCTIONS OF THE EXACT LINEAR EQUATIONS OF MOTION AND THE LINEAR ORBIT PARAMETERS}

The problem now is, given the exact linear equations of motion, Eq. (2), how does one define the linear orbit parameters $\beta, \alpha, \gamma, \psi, v$ and the emittance $\epsilon$, and what are the relationships that hold between them. To do this, one has to repeat the well known treatment of the linear orbit parameters, and see where the definitions and relationships change for the exact equations. The treatment given below is believed to reduce the amount of algebraic manipulation required, and makes few assumptions about the $A_{i j}$ coefficients in the linear equations.

For the $x$ motion, the linear equations are written as

$$
\begin{aligned}
\frac{d x}{d s} & =A_{11} x+A_{12} p_{x} \\
\frac{d p_{x}}{d s} & =A_{21} p_{x}+A_{22} x
\end{aligned}
$$

The transfer matrix $M\left(s, s_{0}\right)$ obeys

$$
\begin{aligned}
x & =M\left(s, s_{0}\right) x_{0} \\
x & =\left(\begin{array}{c}
x \\
p_{x}
\end{array}\right) \\
\frac{d}{d s} M & =A M
\end{aligned}
$$

One may note that the symbol $x$ is used in 2 different ways. The meaning of $x$ should be clear from the context. The matrix $M$ is symplectic as the equations of motions are derived from a hamiltonian. [1,3] Thus

$$
\begin{aligned}
M \bar{M} & =I \\
\bar{M} & =\tilde{S} \tilde{M} S \\
S & =\left(\begin{array}{cc}
0 & 1 \\
-1 & 0
\end{array}\right), \quad I=\left(\begin{array}{ll}
1 & 0 \\
0 & 1
\end{array}\right)
\end{aligned}
$$

S is the transverse of $S$. Also $|M|=1 ;|M|$ is the determinant of $M$.

The one period transfer matrix is defined by

$$
\hat{M}(s)=M(s+L, s)
$$

where $L$ is the period of the $A_{i j}$ in Eq. (4). One can show that $\hat{M}(s)$ and $\hat{M}\left(s_{0}\right)$ are related by

$$
\hat{M}(s)=M\left(s, s_{0}\right) \hat{M}\left(s_{0}\right) M\left(s_{0}, s\right)
$$


The eigenfunctions and eigenvalues of $\hat{M}(s)$ are defined by

$$
\begin{aligned}
\hat{M}(s) x & =\lambda x, \\
|\hat{M}-\lambda I| & =0, \\
\lambda^{2}-\left(m_{11}+m_{22}\right) \lambda+1 & =0
\end{aligned}
$$

where $m_{i j}$ are the elements of $\hat{M}$, and using $|\hat{M}|=1$.

Eqs. (9) shows that the two eigenvalues $\lambda_{1}, \lambda_{2}$ obey

$$
\lambda_{1} \lambda_{2}=1
$$

and for stable motion, $|\lambda|=1$ and $\lambda_{2}=\lambda_{1}^{*}$, and we can write

$$
\lambda_{1}=\exp (i \mu)
$$

Given the eigenfunction at $s_{0}, x_{1}\left(s_{0}\right)$ one can find the eigenfunction or any other point $s$ using

$$
x_{1}(s)=M\left(s, s_{0}\right) x_{1}\left(s_{0}\right),
$$

and $x_{1}(s)$ has the same eigenvalue $\lambda_{1}$. This follows from Eq. (9), using Eq. (8) to relate $\hat{M}(s)$ and $\hat{M}\left(s_{0}\right)$. Also $x_{1}(s)$ obeys the linear equations of motion,

$$
\frac{d}{d s} x_{1}=A x_{1},
$$

which follows from Eq. (12) and Eq. (5). One can show that

$$
x_{1}(s) / \lambda_{1}^{s / L}=f_{1}(s),
$$

where $f_{1}(s+L)=f_{1}(s)$. This follows from

$$
\begin{aligned}
f_{1}(s+L) & =x_{1}(s+L) / \lambda_{1}^{s / L+1} \\
& =\hat{M}(s) x_{1}(s) / \lambda_{1}^{s / L+1}=x_{1}(s) / \lambda_{1}^{s / L}
\end{aligned}
$$

Thus, one can write

$$
\begin{aligned}
x_{1}(s) & =\exp (i \mu s / L) f_{1}(s) \\
f_{1}(s+L) & =f_{1}(s)
\end{aligned}
$$

Eq. (15) can be rewritten as

$$
\begin{aligned}
x_{1}(s) & =\beta(s)^{1 / 2} \exp (i \psi) \\
\psi(s) & =\mu s / L+g_{1}(s) \\
g_{1}(s+L) & =g_{1}(s), \quad \beta(s+L)=\beta(s)
\end{aligned}
$$

Eq. (16) defines the beta functions, $\beta(s)$, except for a normalization multiplyer, for the eigenfunction $x_{1}(s)$. The normalization multiplyer will be defined below. It will be shown first that $\psi$ and $\beta$ are related. To find this relation, one uses the Lagrange invariant [1]

$$
W=\tilde{x}_{2} S x_{1}
$$

where $x_{1}, x_{2}$ are two solutions of the equations of motion. Eq. (17) corresponds to the Wronskian in the treatment of the approximate equations of motion. For $x_{1}$ and $x_{2}$, we use the two eigenfunctions $x_{1}$ and $x_{2}=x_{1}^{*}$.

$$
x_{1}=\left(\begin{array}{c}
x_{1} \\
p_{x 1}
\end{array}\right)
$$

For $x_{1}$ one uses Eq. (16) and for $p_{x 1}$ one finds from the equations of motion

$$
\begin{aligned}
p_{x 1} & =\frac{1}{A_{12}}\left(\frac{d x_{1}}{d s}-A_{11} x\right) \\
W & =x_{2} p_{x 1}-p_{x 2} x_{1} \\
W & =\left[x_{2} \frac{d x_{1}}{d s}-\frac{x_{1} d x_{2}}{d s}\right] \frac{1}{A_{12}} \\
W & =\frac{2 i}{A_{12}} \beta \frac{d \psi}{d s}
\end{aligned}
$$

The beta function $\beta$ is normalized by normalizing the eigenfunctions so that

$$
W=\tilde{x}_{1}^{*} S x_{1}=2 i
$$

which gives

$$
\frac{d \psi}{d s}=\frac{A_{12}}{\beta}
$$

Eq. (22) replaces the familiar result $d \psi / d s=1 / \beta$ which is obtained when $A_{12}=1$. From Eq. (22) one can find a result for the tune. Using $2 \pi v=\psi(C)-\psi(0)$ where $C$ is the circumference of the accelerator, one finds

$$
\nu=\frac{1}{2 \pi} \int_{0}^{C} d s \frac{A_{12}}{\beta}
$$

From Eq. (19) we now find for $p_{x 1}$,

$$
\begin{aligned}
p_{x 1} & =\frac{1}{\beta^{1 / 2}}(i-\alpha) \exp (i \psi) \\
\alpha & =\frac{1}{A_{12}}\left(-\frac{1}{2} \frac{d \beta}{d s}+A_{11} \beta\right)
\end{aligned}
$$

Eq. (25) provides the new definition for the $\alpha$ parameter, which replaces the familiar result $\alpha=-\frac{1}{2} d \beta / d s$. At this point the definition of $\alpha$ may seem arbitrary. It will be seen to be the convenient definition of $\alpha$ when the emittance and transfer matrix are considered.

The eigenfunctions can now be written as, using Eq. (16) and Eq. (25),

$$
\begin{aligned}
& x_{1}=\left[\begin{array}{l}
\beta^{\frac{1}{2}} \\
\beta^{-\frac{1}{2}}(-\alpha+i)
\end{array}\right] \exp (i \psi) \\
& x_{2}=x_{1}^{*}
\end{aligned}
$$

For the results for the emittance and transfer matrix, see [2].

\section{DIFFERENTIAL EQUATIONS FOR THE LINEAR ORBIT PARAMETERS}

This section finds differential equations for $\beta$, and $\eta$

\section{A. Second Order Differential Equation for $x$}

From the first order differential equation for $x, p_{x}$, Eq. (4), one can eliminate $p_{x}$ to find a second order equation for $x$. See [2] for details

$$
\frac{d}{d s}\left(\frac{1}{A_{12}} \frac{d x}{d s}\right)+x\left(-A_{21}-\frac{d}{d s}\left(\frac{A_{11}}{A_{12}}\right)-\frac{A_{11}^{2}}{A_{12}}\right)=0
$$

It has been assumed that $A_{11}=-A_{22}$. 


\section{B. Differential Equation for $\beta$}

To find a differential equation for $\beta$, into Eq. (27) for $x$ put the eigenfunction

$$
\begin{aligned}
& x=b \exp (i \psi) \\
& b=\beta^{1 / 2}
\end{aligned}
$$

We find then, see [2] for details,

$$
\frac{d}{d s}\left(\frac{1}{A_{12}} \frac{d b}{d s}\right)-\frac{A_{12}}{b^{3}}+b\left(-A_{21}-\frac{d}{d s}\left(\frac{A_{11}}{A_{12}}\right)-\frac{A_{11}^{2}}{A_{12}}\right)=0
$$

Eq. (29) is a second order differential equation for $b=\beta^{1 / 2}$. It can be compared to the result found when $A_{12}=1$ and $A_{11}=0$,

$$
\frac{d^{2} b}{d s^{2}}-\frac{A_{12}}{b^{3}}=0
$$

\section{Differential Equation for $\eta$}

$\eta$ and $x$ are related by

$$
x=b \eta, \quad b=\beta^{1 / 2}
$$

In the differential equation for $\eta$ the independent variable is $\psi$ or $\theta$ which are related to $s$ by

$$
\begin{aligned}
d \psi & =A_{12} \frac{d s}{\beta} \\
d \theta & =A_{12} \frac{d s}{v \beta}
\end{aligned}
$$

We find $d x / d s$ and $d\left(A_{12}^{-1} d x / d s\right) / d s$ which are then substituted into Eq. (27) to get the equation for $\eta$, using Eq. (29) to eliminate derivatives of $b$. This gives, see [2] for details,

$$
\frac{d^{2} \eta}{d \theta^{2}}+v^{2} \eta=0
$$

The differential equation for $\eta$ is unchanged.

\section{PERTURBATION THEORY USING THE DIFFERENTIAL EQUATION FOR $\eta$}

The equation for $\eta$, Eq. (33) is often used as a starting point in finding the effects of a perturbing field. The particle coordinates are measured relative to a reference orbit which is the particle motion in a known magnetic field with components $B_{i}$. The exact equations of motion can then be written as

$$
\frac{d x_{i}}{d s}=\sum_{j} A_{i j} x_{j}+f_{i} \quad i=1,4, j=1,4
$$

where the $f_{i}$ includes all the terms not included in $\sum A_{i j} x_{j}$. These include terms due to fields not included in the reference field $B_{i}$, which may be referred as $\Delta B_{i}$, and nonlinear terms due to the terms in the exact equations of motion that do not depend on $B_{i}$.

One can see from the exact equations of motion, that the contributions to $f_{i}$ which depend explicitly on $\Delta B_{i}$, when $\Delta B_{s}=0$, are given by

$$
\begin{aligned}
& f_{2}=\frac{1}{B \rho}(1+x / \rho) \Delta B_{y} \\
& f_{4}=-\frac{1}{B \rho}(1+x / \rho) \Delta B_{x}
\end{aligned}
$$

Repeating the above derivation of Eq. (33) for $\eta$, including the $f_{i}$ terms, one finds the $\eta$ equation for the $x$-motion

$$
\begin{aligned}
\frac{d^{2} \eta}{d \theta^{2}}+v_{x}^{2} \eta & =\frac{v_{x}^{2} \beta_{x}^{3 / 2}}{A_{12}} f_{x} \\
f_{x} & =f_{2}+\frac{A_{11}^{2}}{A_{12}} f_{1}+\frac{d}{d s}\left(\frac{f_{1}}{A_{12}}\right) \\
d \theta & =A_{12} \frac{d s}{v_{x} \beta_{x}}
\end{aligned}
$$

A similar equation can be found for the $y$ motion,

$$
\begin{aligned}
\frac{d^{2} \eta}{d \theta^{2}}+v_{y}^{2} \eta & =\frac{v_{y}^{2} \beta_{y}^{3 / 2}}{A_{34}} f_{y} \\
f_{y} & =f_{4}+\frac{A_{33}^{2}}{A_{34}} f_{3}+\frac{d}{d s}\left(\frac{f_{3}}{A_{34}}\right)
\end{aligned}
$$

For the case of a gradient perturbation

$$
\Delta B_{y}=-G x
$$

one can use Eq. (36) to find the change in $v_{x}, \Delta v_{x}$. One finds

$$
\Delta v_{x}=\frac{1}{4 \pi} \int d s \beta_{x} \frac{G}{B \rho}
$$

This well known result for $\Delta v_{x}$ is not changed by using the exact linear equations.

\section{References}

[1] E.D. Courant and H.S. Snyder, Theory of the Alternating Gradient Synchrotron, Annals. of Physics, Vol. 3, p. 1 (1958).

[2] G. Parzen, Linear Orbit Parameters for the Exact Equations of Motion, BNL Report AD/RHIC-127, BNL-60090 (1994).

[3] F.T. Cole, Notes on Accelerator Theory, MURA report, TN 259 (1961). 